\title{
Implicancias de la nueva normalidad por el Covid-19 en el sistema educativo peruano
}

\section{Implications of the new normality by Covid-19 on the Peruvian educational system in the Peruvian educational system}

DOI: $10.46932 /$ sfjdv2n5-001

Received in: March 1st, 2021

Accepted in: May 30th, 2021

\section{Dr. Elvis Maximo Alvarez Torres}

Doctor y Postdoctorado en Ciencias de la Educación, Magister en Educación Tecnológica, Licenciado en Educación en la especialidad de Telecomunicaciones e Informática todos en la Universidad Nacional de Educación; Segunda Especialidad en Gestión de la Educación en la Universidad Peruana Cayetano

Heredia, Actualmente Docente de la Escuela Superior de Guerra Aérea, Docente de pregrado y postgrado en la Universidad Nacional de Educación, Docente de pregrado y Postgrado de la Policía

Nacional del Perú y docente de postgrado en el Instituto Científico Tecnológico del Ejército

\section{MBA Nadia Yanira Maycock Pérez}

MBA por la Universidad de Piura, Maestro en Doctrina y Administración Aeroespacial por la Escuela Superior de Guerra Aérea, Bachiller en Administración de Empresas por la Universidad Federico Villareal, Programa Avanzado de Administración de Empresas en la Escuela de Negocios ESAN, Curso Básico en Derecho Internacional Humanitario y Derecho Internacional de los Derechos Humanos, Gestión Estratégica de Recursos Humanos en la Escuela Superior de Guerra Aérea, Programa de Especialización en la Universidad de Lima, Especialización en Gestión por Procesos en SERVIR; Curso para Oficiales Militares Femeninas con intención de participación en las Misiones de Paz de las Naciones Unidas (ONU) La Haya - Holanda.

\section{Arq. Katherine Jazmin Alvarez Cardenas}

Arquitecta por la Universidad Ricardo Palma (URP). diplomado en diseño de espacios comerciales y corporativos en la UCAL. Especialista en diseño de interiores. Experiencia en diseño y supervisión de acabados en proyectos de arquitectura residencial, educativa y comercial. Fundadora de Manzana L Studio (2017)

\section{Bryan Samir Alvarez Cardenas}

Facultad de Ciencias de la Comunicación; Universidad de Lima (estudiante)

\section{RESUMEN}

El sistema educativo peruano se encuentra en crisis consecuencia de la pandemia del Covid-19. Los protocolos informáticos (Audio/Video) a distancia forzados en el 2020 se vienen replicando con ligeras mejoras en el 2021 para solucionar el problema del distanciamiento social en el sistema educativo estatal, lo que se asumió de manera similar en el sistema educativo privado. El presente estudio es una investigación cualitativa exploratoria basado en la "observación participante", se utilizó el método de revisión analítica, seleccionando experiencias, analizando la teoría y la praxis educativa. Tiene como objetivo, identificar la práctica docente y estudiantil en situación de pre y post-pandemia. Desde la perspectiva docente, un pequeño grupo contaba con competencias digitales; pero, la mayoría se mantuvo alejada de las TICs improvisando su aplicación en el 2020; Los estudiantes, no cuentan con un plan estructurado, algunos no tienen computadoras, otros no tienen internet o tarifa plana utilizando celulares; la adaptación por parte de los estudiantes es complicada; algunos en clases solo marcan asistencia, pues se registran y no están pendientes de las sesiones, otros argumentan fallas inexistentes; esta situación, es probable que se agudice 
en el 2021. El presente trabajo concluye que el Covid-19 ha incrementado metas de atención en las instituciones educativas públicas; la teoría educativa ha cambiado y es necesario plantear un nuevo paradigma educativo.

Palabra clave: Educación en el Perú, TICs y desarrollo docente, educación en pandemia, formación docente en TICs, tecnología y educación.

\begin{abstract}
The Peruvian educational system is in crisis as a result of the Covid-19 pandemic. The computer protocols (Audio/Video) at a distance forced in 2020 are being replicated with slight improvements in 2021 to solve the problem of social distancing in the state educational system, which was assumed in a similar way in the private educational system. The present study is an exploratory qualitative research based on "participant observation", the analytical review method was used, selecting experiences, analyzing the theory and educational practice. Its objective is to identify the teaching and student practice in a pre- and post-pandemic situation. From the teaching perspective, a small group had digital skills; But, the majority stayed away from ICTs, improvising their application in 2020; The students do not have a structured plan, some do not have computers, others do not have internet or a flat rate using cell phones; adaptation by students is complicated; some in classes only mark attendance, since they are registered and are not pending the sessions, others argue non-existent failures; This situation is likely to worsen in 2021. This study concludes that Covid-19 has increased care goals in public educational institutions; educational theory has changed and it is necessary to propose a new educational paradigm.
\end{abstract}

Keywords: Education in Peru, ICTs and teacher development, pandemic education, teacher training in ICTs, technology and education.

\title{
1 INTRODUCCIÓN
}

El sistema educativo peruano se encontraba en una grave crisis antes de la pandemia, agudizándose con la pandemia provocada por el Covid-19 que llevó al distanciamiento social y confinamiento domiciliario a todo el pueblo peruano a partir de marzo del 2020, las dificultades para el pago de las mensualidades en las instituciones educativas privadas así como la problemática de conectividad de datos llevaron a una deserción masiva, los mismos que se integraron al sistema educativo público en el año 2020 y se han mantenido en el presente año 2021 adecuándose al aprendizaje remoto. Se desconoce cuánto tiempo durará la pandemia y qué hará el Ministerio de Educación para resolver la atención presencial de “110,405 estudiantes que se trasladaron al sistema público" (gestion.pe, 2020) no habiéndose previsto una ampliación de la infraestructura así como, las metas de ocupación docente que al trabajar en vía remota aún no se efectiviza dicha necesidad; por otro lado, es preocupante también la problemática de los logros de aprendizaje debido a que no existe un adecuado control de participación y en el año 2020 aprobaron todos los matriculados sin siquiera demostrar asistencia, consecuencia de la justificación por dificultades en el acceso, falta de computadoras, falta de conectividad o limitaciones en el plan de datos, que se replicará en el 2021. Esta situación proyecta la investigación a indagar la situación del sistema educativo nacional a la nueva normalidad, conocer la situación formativa a nivel de educación básica, hasta llegar a la pandemia en el año 2020 y su continuidad en el año 2021; analizando los cambios provocados por la pandemia Covid-19. 


\section{MATERIALES Y METODOLOGÍA}

El presente trabajo es un estudio cualitativo exploratorio que "tiene como propósito examinar un fenómeno o problema de investigación nuevo o poco estudiados, sobre el cual se tiene muchas dudas o no se ha abordado antes" (Hernández-Sampieri y Mendoza, 2019, p. 106); y "la observación es un procedimiento empírico básico que tiene como objeto indagar averiguar y entender los hechos y los datos” (Bunge, 1972 p.717). Asimismo, Crisólogo (1994) nos explica los tipos de observación científica, relacionadas al estudio exploratorio: (1) La observación no estructurada; que se desarrolla con espontaneidad y sin el adecuado control del proceso y las percepciones del observador. (2) La observación sistemática o estructurada; que establece objetivos delimitando el campo de observación, escogiendo los aspectos más relevantes, estableciendo instrumentos y registrando contenidos de manera precisa y responsable. (3) La observación no participante; donde el observador toma contacto con el grupo de estudio permaneciendo ajeno a la situación observada; y, (4) la observación participante; donde el observador permanece dentro del grupo, asumiendo roles; estableciéndose para nuestra investigación en este último en vista que los autores van a relatar sus propias situaciones dentro del contexto de la investigación. (pp. 94-96)

La educación en la era pre-Covid

Muchos nos lamentamos del comportamiento ciudadano para la elección de representantes probos y elevado carácter ético en la Presidencia de la República, Congreso de la Republica, Gobiernos Regionales y Locales; pero no analizamos el fondo del asunto, conocer cómo es que llegamos al punto en el cual, el ciudadano común y corriente no tiene capacidad de decidir y es manipulado por un táper ${ }^{1}$ vacío en época de elecciones o una publicidad sin sentido, en vista que es necesario incorporar de manera transversal la ética y la política a las prácticas educativas y pedagógicas (Pino \& Euscátegui, 2021); Arnove nos explica: "El Estado continúa condicionado por la políticas económicas y sociales neoliberales que se aplican en los países de la región con el objeto de lograr acceso al capital y a los mercados internacionales..." (2004, p. 47) siendo incomprensible entender que en pleno Siglo XXI seguimos viviendo como en los señoríos feudales y nuestro país recibe el dictado de nuestras reglas de conducta por parte de las transnacionales, "Perú, a pesar de haber mejorado, no ha tenido buenos resultados en la prueba PISA. (...) el Ministerio de Educación debe asumir como reto, generar estrategias que mejoren el rendimiento académico”. (Marín, 2017; recuperado de Contreras et al, 2021). En ese sentido, Arnove nos explica que nuestras "políticas de ajuste estructural [son] recomendadas por el Banco Mundial, el Fondo Monetario Internacional y las Agencias Nacionales de Asistencia Técnica como la USAID (United States Agency for International Development) afectando la práctica de la educación” (2004, p. 47).

\footnotetext{
${ }^{1}$ Recipiente con cierre hermético, que se usa para guardar o llevar alimentos (DRAE, 2021)
} 
En América Latina, las políticas y programas educativos están limitados en vista que los Ministros de Educación se ven obligados a seguir las políticas de las agencias de cooperación (Arnove, 2006, pp.52,54), quienes aconsejan “disminuir la intervención del Estado en el proceso educativo y aumentar la representación del sector privado y la aplicación de los principios del libre mercado en los sistemas educativos del tercer mundo" (Berman,1992, p. 69); por ende, la división de estudiantes por clases sociales; los padres de familia de las clases media y alta trasladan a sus hijos a instituciones privadas, las clases populares se mantienen en el sistema de educación pública, que, sin el apoyo de la clase media carecen de influencia para promover la calidad del sistema educativo (Arnove 2006, p. 55). La alta interacción estudiantil tanto en el salón de clases, en la hora de recreo y fuera de horas de clases, aunado a la comunicación permanente por redes sociales. Los docentes y estudiantes desconocen la capacidad de utilizar las TICs.

La Infraestructura educativa se mantiene por años como es el caso del Colegio Julio C. Tello ubicado frente a la Plaza de Armas (El pueblito) del Distrito de San Juan de Lurigancho en Lima que, siendo la oficina de coordinación principal de Defensa Civil de la Red educativa, cuenta con infraestructura declarada inhabitable. Los docentes se agrupan para el dictado de cursos autoformativos en vista que la UGEL $05^{2}$ no permite el contrato de especialistas de alta competividad con cargo a recursos directamente recaudados, siendo necesario además que los docentes enfoquen una orientación histórico cultural para desarrollar la personalidad de los estudiantes (Cánovas, 2021). El transporte de datos se obtiene mediante contrato con Telefónica del Perú que asigna anchos de banda que garantizan únicamente el $10 \%$ del volumen contratado provocando lentitud en el servicio y perjudica la asignación de Internet en los laboratorios. En el sistema rural, en muchas localidades no existe conexión de datos. La economía familiar conlleva a un bajo nivel de nutrición estudiantil que influye directamente en el logro de los aprendizajes. El gobierno no presupuesta con el estándar de Latinoamérica, la infraestructura educativa en el sistema rural es aún más grave.

\footnotetext{
${ }^{2}$ Unidad de Gestión Educativa Local de San Juan de Lurigancho, Lima-Perú, unidad de control de las instituciones públicas de la zona.
} 


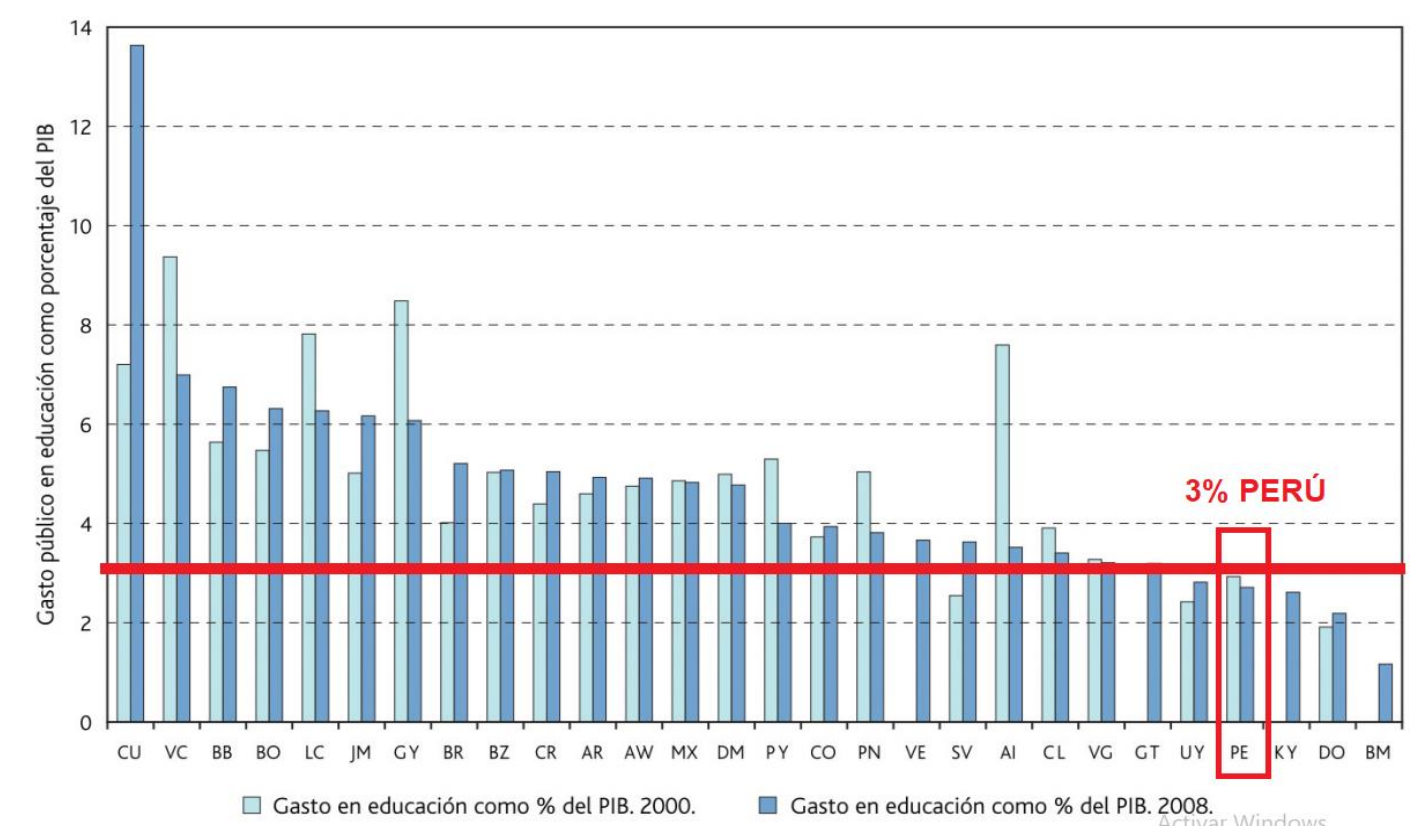

Fuente: Gasto público en la educación de América Latina-UNESCO (Toledo, Botero \& Guzmán, 2014)

Las nuevas tecnologías de la información y comunicación

El desarrollo de la tecnología se dio inicio como consecuencia de la generalización del uso de la corriente eléctrica, en 1830 Michael Faraday invento el primer dinamo o generador eléctrico (areatecnologia.com, 2021), se generalizó con el invento de la bombilla incandescente por parte de Thomas Alva Edison en 1878. Seguidamente, el descubrimiento del efecto Edison (Wikipedia.org, 2021) llevando hasta nuestros días el potencial de desarrollo de la tecnología. Como consecuencia del descubrimiento del transistor (Transfer Resistor) en 1947, por parte de los Físicos William Shockley (Premio Nobel de Física en 1956) Walter Brattain, y John Bardeen, entramos a la era de la electrónica. Este desarrollo; que se aceleró a partir del Siglo XX nunca fue apareado con el sistema educativo, de tal manera que hasta antes de la pandemia los sistemas tecnológicos correspondían al uso de una computadora así como sus aplicaciones básicas enfocado generalmente a sus aplicaciones de oficina, desligándose totalmente de todo el desarrollo acelerado tanto del software como Hardware que ha avanzado desmesuradamente en la actualidad comenzando únicamente con el tema de contenidos, se ha desarrollado sistemas de almacenamiento extraordinarios denominados data center que puede contener toda la información de la humanidad y cuyo almacenamiento se encuentra replicado con discos espejos en diferentes lugares del mundo (world wide web) a fin de contener una probable pérdida de datos conllevando esta situación a la casi extinción de las bibliotecas físicas así como las limitaciones que ella conlleva; generalizándose las bibliotecas digitales que actualmente se encuentran disponibles en la mayor parte del mundo y que requieren únicamente una conectividad de datos para tener acceso a internet. 
El desarrollo de microprocesadores ha avanzado hasta la nanotecnología contando en la actualidad con celulares con la misma o superior potencia (caso $\mathrm{ARM}^{3}$ ) y acceso que las computadoras, conllevando a la democratización del conocimiento que antes estaba limitado únicamente a las altas clases sociales, los mismos que buscaron mantener y controlar mediante el control de acceso pero que las facilidades de conectividad han quebrado este impedimento.

En relación a los avances tecnológicos se encuentran aún vacíos o en etapa germinal, tenemos: El pensamiento computacional que cubre la necesidad de creación de códigos computacionales que puedan servir como software de programación para diversas aplicaciones basado únicamente en códigos de afirmación o negación (si-if); La realidad aumentada o realidad virtual nos puede llevar a situaciones reales pero de acceso físico imposible y con acceso y aproximación al nivel micro, o macro dependiendo de las necesidades del usuario. Los juegos serios, videojuegos o gamificación en vista que el docente considera los juegos como algo negativo no entendiendo que la modernidad exige configurar procedimientos buscando aprendizajes con herramientas divertidas para el discente, implicando que el juego puede configurar una mejor calidad en el logro de aprendizajes; la gamificación puede desarrollar procedimientos complejos explorando el interés en el juego y logrando el desarrollo de estrategias imposibles mediante los métodos de enseñanza actuales. Desde hace 90 años $^{4}$ han existido los sistemas de educación a distancia y hace 20 años se han implementado las plataformas para la enseñanza y aprendizaje mediante el uso de las tecnologías de la información y comunicación que han estado implementados sin uso en las universidades y avanzando aisladamente en las escuelas de posgrado pero nunca llegaron al pregrado contando en estos momentos con un desarrollo amplio y complejo que complementan tanto contenidos, presentaciones, videos y evaluaciones remotas, que se pueden concatenar con el sistema presencial a fin de potenciar el sistema docente. Finalmente, existe un avance en el sistema universitario con el uso y aplicación de los sistemas robóticos generalmente aunado a microcontroladores como Arduino ${ }^{5}$, pero que se trabaja con algunos docentes que tienen interés personal pero que no se encuentra estandarizado dentro del sistema educativo.

\footnotetext{
${ }^{3}$ Los procesadores ARM son la opción ideal, ya que utilizan un método de procesamiento simplificado y que consume menos energía. Esto se representa en el mismo nombre ARM, que significa «Advanced RISC Machine» o máquina RISC avanzada, donde RISC significa «Reduced Instruction Set Computer». (hardzone.es https://hardzone.es/tutoriales/componentes/procesador-arm/)

${ }^{4}$ En el año de 1920 Ralph Hemphill fundó la primera escuela HEMPHILL SCHOOLS en Vancouver, Canadá; en ella se impartían cursos, en forma tradicional, de mecánica diesel. Hacia 1930 abrió escuelas en los Estados Unidos, en donde cambió su método de enseñanza al de la educación a distancia. (http://www.hemphillschools.com/geninfo.html)

${ }^{5}$ Arduino es una plataforma de creación de electrónica de código abierto, basada en hardware y software libre, flexible y fácil de utilizar para los creadores y desarrolladores. Esta plataforma permite crear diferentes microordenadores de una sola placa (https://www.xataka.com/basics/que-arduino-como-funciona-que-puedes-hacer-uno)
} 
Teorías pedagógicas que avalan el aprendizaje con TICs

La escolástica nace en el siglo XIII con Santo Tomas de Aquino es un método de trabajo intelectual donde todo pensamiento debía someterse al principio de autoridad, y la enseñanza se limitaba en el principio a la reiteración de los textos clásicos, la Biblia (principal fuente de conocimiento) fue el incentivó, el razonamiento y la especulación pues suponía la adaptación a un riguroso sistema lógico y un estructurado esquema del discurso que debía ser capaz de exponerse a refutaciones y defensas (Wikipedia.org, 2021). Actualmente, las teorías de aprendizaje mantienen la noción de que el conocimiento es un objetivo alcanzable a través del razonamiento o la experiencia. El conductismo, el cognitivismo y el constructivismo, intentan evidenciar cómo una persona aprende. El conductismo, como lo señala Gredler, establece que el aprendizaje es incognoscible, no podemos entender qué ocurre dentro de la mente de una persona (la "teoría de la caja negra"). Expresándose como un conjunto de teorías que hacen tres presunciones del aprendizaje: El comportamiento observable es importante, El comportamiento se enfoca en estímulos y respuestas y El aprendizaje tiene que ver con el cambio del comportamiento (2001). El cognitivismo toma el modelo computacional del procesamiento de la información donde el aprendizaje es un proceso de entradas, administradas en la memoria de corto plazo, y codificadas para su recuperación a mediano y largo plazo, la existencia de tres sistemas básicos de representación presentes en la cognición humana: La representación enactiva (representación de las cosas mediante la reacción de las personas), la representación icónica, mediante imágenes de objetos o eventos, y la representación simbólica, usando sistemas formales como el lenguaje (Camargo \& Hederich, 2010).

El conductismo y el cognitivismo dan el conocimiento externo al aprendiz y el aprendizaje como al acto de aprehender (capturar) el conocimiento. El constructivismo asume que los aprendices no son simples recipientes vacíos para ser llenados con conocimiento; sino que seleccionan y persiguen su propio aprendizaje mientras tratan de comprender sus experiencias (Driscoll, 2000, p. 376). Valdez propone el socio-constructivismo, como la teoría educativa que facilita el uso de las TICs, tanto en la modalidad presencial como virtual; esta teoría facilita la interacción social y el trabajo grupal que se requiere para generar conocimiento y aprendizaje en un ambiente educativo mediado por la tecnología; ninguno de los preceptos desarrollados por las teorías educativas son excluyentes en la educación mediante TICs; del conductismo, se puede tomar el modelo de evaluación para identificar los avances del aprendizaje; del cognitivismo, el concepto de esquemas y procesos mentales que permite la modificación del conocimiento y el establecimiento de significados; del constructivismo, el conocimiento previo como andamiaje para el nuevo conocimiento, en donde el alumno reordena y adapta los conocimientos previos; y, el socio constructivismo reconoce la 
importancia del entorno social en el proceso de adquisición del conocimiento con la influencia de la sociedad, la familia, los amigos, la escuela y el trabajo. (2012).

Robles \& Zambrano señalan la incorporación de las TICs en el proceso de enseñanzaaprendizaje, permitiendo la interacción docente-estudiante como herramienta didáctica que consolida el aprendizaje significativo, siendo importante que los docentes incluyan estas tecnologías en la enseñanza de los estudiantes; el nivel de uso de las tecnologías en las aulas de clase, depende de las habilidades y actitudes del maestro. Las prácticas académicas basadas en nuevas tecnologías para el desarrollo de ambientes creativos de aprendizaje, constituye una propuesta relevante y permiten un aprendizaje más efectivo. (2020); lamentablemente Ames (2019, como se citó en Quispe y Huamán, 2021) "sostiene que [en] algunas unidades educativas no se hace uso de las TIC, debido a las insuficientes competencias digitales del docente".

El Conectivismo, una nueva propuesta teórica educativa basada en las TICs

Siemens señala que, el Conectivismo reconoce que el aprendizaje en la vida real es caótico y complejo. Las aulas de clase emulan la "ambigüedad" de este aprendizaje preparando al estudiante para el aprendizaje a lo largo de la vida (2004). Siemens muestra una mirada diferente al sistema educativo actual donde "La tubería es más importante que su contenido", y explica que, debido a la caducidad de las tecnologías, necesitamos aprender procedimientos para que ningún contenido sea eminentemente histórico. Por lo tanto, necesitamos que, la teoría de aprendizaje nos explique la acción procedimental en el sitio de la aplicación para poder recabar los contenidos que fluyen a cada instante. Esto nos deja atónitos en vista que, por siempre desde la escolástica, se nos ha explicado que más importante es "cuanto hemos pescado hoy", por encima de "como hemos pescado" Sin embargo, el conocimiento fluye en dimensiones estratosféricas (Big Data) y se va sin siquiera haberlo observado, siendo necesario la habilidad de conectarse mediante grandes autopistas de datos o sistemas de hardware de gran capacidad de procesamiento que corresponden a mejorar la habilidad para el multiprocesamiento. A medida que el conocimiento crece y evoluciona, se amplían mas las perspectivas y las necesidades de contenido se hacen ilimitadas.

El Conectivismo es la propuesta de un nuevo paradigma de aprendizaje que reconoce la gran capacidad de las nuevas tecnologías y su aporte en una sociedad en donde el aprendizaje de contenidos ha dejado de ser una actividad interna e individual en vista que las nuevas herramientas son extensiones de nuestro cerebro y se potencian en múltiples dimensiones tanto físicas (data center) como biológicas (humanas) dependiendo de la capacidad de conectividad con dichas herramientas. Los agentes educativos han sido lentos en reconocer el impacto de estas nuevas herramientas y la pandemia los ha obligado reconocer sus múltiples aplicaciones que ya existían previamente. El Conectivismo provee una mirada 
diferente a las habilidades de aprendizaje donde la conectividad y las capacidades de procesamiento se priorizarán por encima de la acumulación de contenidos. (2004)

Montoya, Parra, Lescay, Cabello \& Coloma señalan que las TICs propician un cambio significativo en el orden metodológico y didáctico, surgiendo modalidades como el e-learning y el blearning que, sustentadas en el Conectivismo, están revolucionando la enseñanza y el aprendizaje significativo y autónomo, (2019). Siemens explica que, en el Conectivismo, el aprendizaje ocurre al interior de ambientes difusos cambiantes que no están bajo control del individuo, siendo la integración de principios como la teoría de caos, redes, complejidad y auto-organización. Este aprendizaje reside fuera de nosotros mediante conjuntos de información especializada, y a través de conexiones donde se aprende más que nuestro estado actual de conocimiento. El Conectivismo implica que la comprensión y las decisiones están basadas en principios que cambian continuamente. La habilidad de distinguir entre la información que importa y la que no resulta vital, y la habilidad de reconocer cuándo una nueva información altera el entorno de una decisión tomada con anterioridad. Dentro de los principios del Conectivismo, el aprendizaje y el conocimiento dependen de la diversidad y el aprendizaje es un proceso de conectar nodos o fuentes especializadas que en la mayoría de los casos residen en dispositivos no humanos. La capacidad de saber más (prospectivo) es más crítica que aquello que ya se sabe; el mantenimiento de las conexiones es imperativa y la habilidad de ver las conexiones es clave; la actualización es prioritaria y la toma de decisiones puede ser correcta hoy y puede estar equivocada mañana. En el Conectivismo las actividades de gestión de la información residen en bases de datos a miles de kilómetros y deben estar conectados en el contexto adecuado para que pueda ser clasificado como aprendizaje. (2004).

\section{Desarrollo formativo en la era Post Covid-19}

a) Primera experiencia; Mi educación fue muy didáctica en las clases presenciales (20102019). Los profesores enseñaban en pizarra acrílica con apoyo de un sistema multimedia; el colegio entregó una tableta para cada estudiante donde contábamos con libros de todo el año escolar en formato pdf y se tomaba apuntes de los datos más trascendentes; las clases de matemática, no cambian mucho, el profesor explica un problema, los alumnos resuelven uno parecido, cada profesor tiene su propia manera, muchos entendían rápido; algunos no entendían, otros se aburrían o estaban distraídos por las largas horas de clases. No obstante, las clases no eran agotadoras, porque compartíamos las dudas entre compañeros y consultábamos al profesor (Alvarez B., 2021).

En marzo 2020, se anunció la pandemia del Covid-19 en todo el mundo. Se cancelaron las clases presenciales, se anunciaron las clases virtuales; ocurrió un cambio radical en la educación por todo el año 2020, más de 5 horas frente a una pantalla, con 2 recesos de 10 y 25 minutos; se notaba un claro 
desconocimiento del sistema por parte de los profesores, tutoras y alumnos, pese a que habíamos trabajado en este tipo de plataformas. Los profesores explicaban de una manera incomprensible y con muchas complicaciones. Algunos de ellos, no contaban con internet permanente. Con el transcurrir del año, se pudo evidenciar que el nuevo sistema educativo no funcionaba, esta modalidad no se había probado hasta la fecha y no era accesible a todos, porque se necesitaba un dispositivo digital para acceder a los contenidos, tener internet y contar con luz eléctrica. Un nuevo sistema educativo no puede ser implementado en pocos días, se necesita hacer pruebas e ir mejorando con el tiempo; sin embargo, debido a la presión de perder el año escolar, el Estado decidió ejecutar este sistema; no obstante, profesores con más de 30 años frente a una pizarra no pueden conectarse con las nuevas tecnologías, los más experimentados, renunciaron a las nuevas aplicaciones. Por otro lado, un porcentaje de alumnos no contaban con internet o algún dispositivo para asistir a las clases virtuales perjudicando su aprendizaje. En mi caso, contaba con laptop e internet, pero a veces me desconectaba por la pérdida de señal de la PC o del profesor. Las clases síncronas se realizaron mediante Google Meet con un Power Point o un video, Las evaluaciones eran mediante Google Classroom donde los profesores colocan formularios; a veces, las clases eran recuperadas fuera de horario o nos enviaban al correo los materiales de clase como notas, videos, respuestas y sesiones grabadas. La Educación Física se realizaba mediante ejercicios en casa fuera del horario de clase, teníamos que subir un video haciendo ejercicios. En las clases de tutoría nos enseñaban valores, el Plan Lector obliga la lectura de 8 libros al año, eran muy aburridos, pero teníamos que leerlos para responder preguntas que nos daban en una ficha (Alvarez B., 2021).

b) Segunda experiencia; El sistema educativo pasa por una grave crisis provocada por el Covid-19. La problemática al finalizar el año 2019 con sus dificultades y potencialidades no se comparan al comportamiento sintomático del 2020 y replicado al 2021. Los sistemas de educación a distancia que han ido avanzando desde el año 2005, las plataformas de videoconferencia rechazados por muchos años, se implementaron inmediatamente para resolver el sistema de enseñanza aprendizaje en el sistema educativo estatal, replicado mejor o peor en el sistema educativo privado; desde la perspectiva docente, un pequeño grupo contaba con experiencia digital; pero, la mayoría están alejados de las TICs, teniendo que improvisar para obtener las competencias básicas y obtener logros de aprendizaje en el año académico 2020 y repetirlo en el 2021. Por el lado del estudiante, no existe un plan estructurado, en vista que la mayoría no cuenta con computadoras o tabletas ${ }^{6}$, otros no cuentan con internet y la falta de una disponibilidad de tarifa plana acaba rápidamente los planes de datos. Los que no tienen computadora, reemplazan su participación mediante celulares, con las dificultades de visualización y capacidad de

\footnotetext{
${ }^{6}$ El Jefe de Estado detalló que 719,000 tablets con servicio de internet móvil estarán destinados a escolares del 4to, 5to y 6to de primaria y de todos los grados de educación secundaria del ámbito rural, así como 123,780 tablets (...) para alumnos del área urbana en situación de pobreza". Hace 10 meses. (Del Águila, 2020 )
} 
respuesta; respecto al transporte de datos, transferencia de contenidos, consumo de datos, acondicionamiento y adaptación de los estudiantes, simplemente marcan presencia apagan su cámara web o comunican ausencia por fallas en la conectividad, situación que se irá repitiendo en el 2021. (Alvarez E., 2021)

\section{RESULTADOS}

Es evidente que los sistemas de enseñanza a distancia tienen una larga existencia, y los sistemas de educación a distancia tienen una vigencia paralela a la enseñanza tradicional los mismos que no necesitaron a la informática para desarrollarse; la teoría educativa se ha ido enmarcado en una corriente continua desde el conductismo, cognitivismo, construccionismo hasta el constructivismo social en el cual nos encontramos actualmente. Todos ellos se enfocan hacia el logro de los aprendizajes del ser humano mediante diferentes estrategias metodológicas. Es generalizada la concepción que las nuevas tecnologías han venido para quedarse y de alguna manera, deben adaptarse al sistema educativo para potenciar los logros de aprendizaje; hasta el momento, solamente logramos su aceptación y aplicación, de facto, como consecuencia de la pandemia mundial del Covid-19. Es probable que, en el 2021, los daños serán sobrepasados, ya que las informaciones recientes, tratan sobre la tercera ola que provocará no solo una crisis económica, sino también social y educativa a nivel mundial. En ese contexto, es necesario reconocer la existencia de graves dificultades en el sistema educativo pre-Covid, y que se agudizará en la era post Covid, en vista que no estamos preparados para el aprendizaje en confinamiento; en tal sentido, los sistemas de educación a distancia, con formato digital existentes desde el año 2005, se plantearon como una alternativa obligatoria para el año 2020, encontrándose una plataforma docente desinformada de los procesos cognitivos, que en su mayoría han utilizado el sistema digital solo como entretenimiento. Es necesario reconocer que dichas debilidades repercutirán dramáticamente en un futuro próximo con ciudadanos que no se adecuarán a los sistemas de aprendizaje virtual y que no toman en consideración las decisiones del supremo gobierno, así como por las debilidades generacionales.

Para obtener logros de aprendizaje, es necesario implementar una teoría educativa contextualizada a las herramientas y aplicaciones de las nuevas tecnologías de la información y comunicación, en las que se incorporen las proyecciones que proponen estas herramientas cuya aceleración es extraordinaria; asimismo, "sólo los países capaces de hacer del conocimiento, la cultura y el progreso técnico el sustento de la inclusión social, del bienestar colectivo y el motor de la competitividad económica tendrán viabilidad en el concierto mundial" (Contreras et al, 2021). Por lo manifestado, se pone en consideración el Conectivismo como un principio teórico que requiere un 
desarrollo epistemológico para que se adecúe con total naturalidad al sistema educativo. Conceptos como la teoría del caos, sistemas de redes, teoría de la complejidad y la auto-organización son de necesaria teorización para lograr una adaptación a nuestro sistema educativo.

\section{DISCUSIÓN}

Buscar la responsabilidad de los graves problemas de la educación peruana a la pandemia del Covid-19 es absolutamente irresponsable, en vista que, como se ha analizado en el capítulo de "la educación en la era pre covid", la bilateralidad del comportamiento del Estado Peruano, es extremadamente evidente. Por un lado, la Constitución Política del Perú en su Art. 17 señala que "La educación inicial, primaria y secundaria son obligatorias [y que] en las instituciones del Estado la educación es gratuita"7 como contrariedad. el Art. 15 indica que "toda persona natural ${ }^{8}$ o jurídica tiene derecho de promover y conducir instituciones educativas (..) fijando la Ley el modo de subvencionar la educación privada en cualquiera de sus modalidades.."(CPP, 1979) fomentando así que las "instituciones privadas sean atractivas para los estudiantes de clase media y alta, ofreciendo clases con grupos más pequeños, mayores facilidades y una atmósfera general que estimula el aprendizaje (...) provocando efectos negativos sobre las escuelas públicas" (Arnove, de Plank et al.; 1994) debiendo entender que las principales experiencias que tienen los estudiantes en la escuela les permite desarrollar sus actitudes y valores, puesto que, los resultados de aprendizaje no dependen únicamente de los procesos de enseñanza, sino de las experiencias en general que ocurren a través de la participación, involucramiento o interacción social (Galindo et al, 2021); y cuando los padres de familia de las clases medias abandonan el sistema de educación pública desaparecen también los defensores más enérgicos de una educación de calidad haciendo que las clases populares se constituyan en la mayoría de la población que carecen de influencia política como para promover la calidad de la educación pública; y, sin el apoyo de la clase media las escuelas comienzan a decaer deteriorándose los sistemas educativos nacionales (Arnove, 2006:55)

Contamos en la educación pública con un sistema meritocrático basado en la Ley de la Reforma Magisterial donde se establecen parámetros para el ascenso cada tres años, lo cual no garantiza la estandarización de las competencias básicas en nuevas tecnologías, lo que nos ha dejado expuestos a las deficiencias tecnológicas de la pandemia del Covid 19. Es innegable que en el 2020 hubo avances forzados y maquillajes para demostrar estabilidad; aunque se ha falseado los reportes de logros aceptando que los matriculados 2020 sean aprobados, pese a que no asistieron a clase alguna. No se consideró si el alumno tenía o no computadora, o la conectividad necesaria. Los estudiantes, lamentablemente, conocían la

\footnotetext{
${ }^{7}$ En las instituciones educativas públicas se han implementado las ASPAFAS (asociaciones de padres de familia) que aportan económicamente para el mantenimiento y la infraestructura, además participan en programas de trabajo y limpieza.

${ }^{8}$ Cualquier persona puede ser promotor de una institución educativa privada, no es requisito contar con un grado académico
} 
decisión de que pasarían de año9 .. Lo que resta es decidir si es posible continuar aprobando a los estudiantes que no asistan en el año 2021, generar un equilibrio calificativo entre estudiantes que se esfuerzan, con los que no asistan. Al ser reconocido el año 2021 como el "Año del Bicentenario del Perú: 200 años de Independencia", debemos reconocer que es necesario tomar decisiones respecto a continuar permitiendo que una considerable cantidad de alumnos aprueben sin estudiar y los docentes cobren sin trabajar con el pleno de sus estudiantes.

Finalmente, señala Daisy Loyola de los especiales de El Comercio, 110,405 estudiantes de educación básica regular han retornado a las escuelas públicas. De "Aprendo en Casa", de los 500 gobiernos locales, 462 reportaron dificultades para acceder a los programas y de ellos 268 solicitaron cobertura de radio, televisión o internet; de tal manera que según el INEI, solo el 5.6\% de la población rural tiene acceso a internet. Lo más lamentable es que a pesar de haberse realizado un contrato anticipado para la compra de tabletas este no se efectivizará, porque no existía la garantía de aval de la empresa y que los planes con AOC y Lenovo aún se encuentran en proceso y las clases comienzan el 19 de abril. Existe también el compromiso de generalizar la conectividad de Internet por medio de satélites, pero eso es una utopía en vista que la transferencia de datos actualmente solo se puede cubrir por fibra óptica y que, a pesar de contar el Perú con el Erbio que es la materia prima fundamental para la fabricación de la fibra óptica, esta, prácticamente se obsequia como impureza a las grandes fábricas de fibra óptica en Canadá, cuando debería ser controlado por el Estado, como cientos de países que controlan sus metales $\operatorname{raros}^{10}$, pero que nuestro país exporta de manera libre y casi gratuitamente.

Conforme a la información de Delsy Loyola, de la Unidad Investigación del diario El Comercio, el Sr. José Luis Díaz del despacho de Gestión Pedagógica del Ministerio de Educación, aseguró que “no podemos hablar de un año perdido" y que se está evaluando una adecuada estrategia para los primeros meses del año 2021; no obstante, nos encontramos en abril del 2021 y se puede evidenciar que este compromiso no se cumplió y tampoco se observan avances respecto a la conectividad, por lo que solo el $5,6 \%$ estudiantes del área rural cuenta con internet el presente año. (Loyola, 2020)

\footnotetext{
${ }^{9}$ El ex ministro de Educación Idel Vexler Talledo admitió (...) la posibilidad de que los escolares (...)que están bajo el sistema de educación a distancia, aprueben el año de manera automática (29-05-2020) https://guik.pe/2020/05/29/vexler-no-se-puede-aprobar-el-ano-escolar-automaticamentel

10 Metales raros es el nombre común de 17 elementos químicos como el Escandio, Itrio, Lantano y 14 elementos que siguen en la tabla periódica, algunos, como el Cerio, el Itrio o el Neodimio son más abundantes que el plomo; y el tulio que es escaso, es más común que el oro 0 el platino. E único que no se halla en forma natural es el promecio porque sus isótopos radiactivos se forman en los reactores nucleares. Hace medio siglo la economía mundial dependía de la madera, hormigón, hierro, cobre, oro, plata y plástico. Actualmente, las innovaciones tecnológicas requieren de tierras raras. La nitidez de la pantalla se debe al Europio. El indio permite el roce de los dedos (touch screen). La información de internet llega a nosotros gracias a la fibra óptica fabricada con erbio; los auriculares llevan imanes de neodimio sustituto de la ferrita, Las tierras raras permiten la miniaturización electrónica (López, 2015).
} 


\section{CONCLUSIONES}

a) El sistema educativo peruano antes del inicio de la pandemia, se encontraba en una crisis provocada por las recomendaciones de los organismos internacionales para la implementación de políticas neoliberales que, a lo largo de los años, han intervenido en el proceso educativo público, promoviendo el incremento educativo del sector privado.

b) No se evidencian mejoras en el sistema educativo en el año 2020 comparativamente con los primeros meses del 2021, salvo la entrega de implementos de bioseguridad que se han quedado en los almacenes de las instituciones educativas en vista que los docentes y estudiantes están realizando trabajo remoto.

c) La pandemia del Covid-19 ha puesto en evidencia la existencia de las nuevas tecnologías de información y comunicación y su relación con los procesos de enseñanza aprendizaje actuales, pero que desde hace mucho tiempo han sido subutilizados, debido a una falta de capacitación generalizada a los docentes públicos a nivel nacional, pero que ha sido de mucha utilidad para el desarrollo de las clases sincrónicas no presenciales y el desarrollo de sesiones de clase en el confinamiento provocado por la pandemia Covid-19.

d) Desde el punto de vista teórico, ni el Ministerio de Educación ni la academia, se han preocupado en establecer el estado del arte de la epistemología educativa en situación de pandemia, por lo que no se ha proyectado investigaciones relacionadas a la teoría pedagógica y la cultura científica. Esto motivó que los docentes se encuentran al desamparo, por lo que debieron retornar al conductismo puro, donde el educador hace lo imposible por mantenerse como director de orquesta en un entorno totalmente desconocido.

e) Se presenta la propuesta pedagógica del conectivismo, aunque en un contexto muy focalizado que requiere un marco ampliatorio, que será objeto de una nueva investigación.

f) Recién se inicia el conocimiento de las potencialidades tecnológicas de nuestro suelo patrio y su preponderancia dentro del contexto de las nuevas tecnologías que implica la protección de los metales raros que es "el nuevo oro" del futuro.

\section{RECOMENDACIONES}

a) Regularizar las metas de ocupación para el retorno a clases en la nueva normalidad equilibrado con las metas de atención que han aumentado sustancialmente consecuencia del traslado de los estudiantes de educación básica del sistema educativo privado al sistema educativo estatal.

b) Establecer estándares básicos en competencias digitales para que el $100 \%$ de los docentes de educación básica puedan trabajar eficientemente en el contexto de las TICs. 
c) Incorporación de las nuevas competencias en el Currículo pedagógico básico, Tecnológico y Universitario a fin de optimizar el uso de las herramientas, como la exploración en las nuevas formas de utilización; así como la Investigacion y adaptación de las nuevas TICs aplicadas a educación.

d) Con la nueva normalidad se hace indispensable la generalización de la conectividad remota debiendo asegurar dicha conectividad tanto del lado del docente como del lado estudiante a nivel nacional. 


\section{REFERENCIAS BIBLIOGRÁFICAS}

Alvarez Cárdenas B.S. (2021); Comunicación Personal, 01 de abril del 2021.

Alvarez Torres E.M. (2021); Comunicación Personal, 01de abril del 2021.

Areatecnologia.com (2021); "Descubrimiento de la electricidad" recuperado de: https://www.areatecnologia.com/electricidad/descubrimiento-de-la-electricidad.html

Arnove, F. R. (2006) La educación en américa Latina: dependencia, subdesarrollo y desigualdad. Barcelona: Fundació CIDOB, ISBN 8487072666. <https://ddd.uab.cat/record/174834> [Consulta: 25 febrero 2021].

Bunge M. (1972) La investigación Científica, su estrategia y su filosofía, Ediciones Ariel S.A.- Esplugues de Llobregat, Barcelona 1969, Deposito Legal B. 20.335-1972, España.

Cánovas Fabelo Lesbia Gertrudis (2021) Preparar al maestro para el cambio educativo Correo: lesbia@rimed.cu; South Florida Journal of Development, Miami, v.2, n. 3 , p. 4459 - 4467 special edition, $\begin{array}{lllllllll}\text { jul. } 2021 \text {. ISSN } 2675 \quad-\quad 5459 ; & \text { DOI: } 10.46932 / \mathrm{sfjdv} 2 \mathrm{n} & 3 & - & 0 & 49\end{array}$ https://southfloridapublishing.com/ojs/index.php/jdev/article/view/600/557

Comisión Económica para América Latina y el Caribe (CEPAL)- Organización de las Naciones Unidas para la Educación, la Ciencia y la Cultura (UNESCO) (2020) en su informe "La educación en tiempos de la pandemia de COVID-19" Secretaria Ejecutiva de la CEPAL, Alicia Bárcena, y Directora de la Orealc/Unesco Santiago, Claudia Uribe.

Contreras Sánchez Nancy Elizabeth, Chachi - Espinoza María Mónica, Ibarguen - Cueva Francis Esmeralda, Raggio - Ramírez Giuliana del Socorro, Menacho - Vargas Isabel \& Alvarez - Huari María Ysabel (2021) Estrategias de aprendizaje y comprensión lectora en estudiantes de educación básica regular del Perú; Correo: elyzabetalex2012@gmail.com, monicachachi@gmail.com; francisibarguen@gmail.com, giulianita2706@gmail.com; isabelmenachov@ gmail.com y alvarezhmariay @ gmail.com; South Florida Journal of Development, Miami, v.2, n.2 , p. 2398 - 2407 apr./jun . 2021. $\begin{array}{lllllllll}\text { ISSN } & 2675 \quad- & 5459 ; & \text { DOI: } & 10.46932 / \operatorname{sfj} d v 2 n & 2 & - & 0 & 99\end{array}$ https://southfloridapublishing.com/ojs/index.php/jdev/article/view/354/337

Contreras Vidal Jorge Luis, Yanay Hidalgo Leonard, Loor Almeida Rafael Antonio, Curbelo Sosa Henry \& Pedraza González Xenia (2021) Formación de una cultura científica a través de tareas para el aprendizaje E - mail: jcontreras@ uclv.cu, yanayhl@ crr.sc.vc.rimed.cu, antonilooralmeida@ hotmail.com; henrycurbelo@umma.com.mx, xeniapedraza@gmail.com; South Florida Journal of Development , Miami, v.2, n.2 , p. 2698 - 2706 apr./jun . 2021. ISSN 2675 - 5459; DOI: 10.46932/sfjdv2n 2 - 123 https://southfloridapublishing.com/ojs/index.php/jdev/article/view/388/361

Crisólogo Arce A. (1994) Conceptos, métodos y modelos de la investigación científica - Manual de introducción a la investigación científica, Ediciones Abedul E.I.R.L. Lima Perú, Jr. Azángaro 1076-Of. 3, Lima 1 Teléf. 426-7234

Del Águila Tuanama E. (2020) Martín Vizcarra aplaza inicio de clases escolares presenciales y anuncia compra de Tablets para estudiantes, Agenda Pais.com. 
Delsi Loyola J. (2020) Educación pública en crisis durante la pandemia. Especial del Diario El Comercio 09 de setiembre del 2020; https://elcomercio.pe/peru/coronavirus-en-peru-educacion-publica-en-crisis-durantela-pandemia-de-covid-19-especial-noticia/

Díaz Dumont, J. R. (2018) Políticas públicas en propiedad intelectual escrita. Una escala de medición para educación superior del Perú. Revista Venezolana de Gerencia, vol. 23, núm. 81, 2018 Universidad del Zulia, Venezuela Revista Venezolana de Gerencia ISSN: 1315-9984 rvgluz@fces.luz.edu.ve; http://www.redalyc.org/articulo.oa?id=29055767006

Driscoll, M. (2000). Psychology of Learning for Instruction. Needham Heights, MA, Allyn \& Bacon.

Galindo - Kimura Magaly Analí, Menacho Vargas Isabel, Huertas Rueda Edith Mercedes \& Valentín Montes Máximo Guillermo (2021) Convivencia sin violencia en una escuela pública del Perú Correo: missmagaki@gmail.com, isabelmenachov@gmail.com, valedocente@gmail.com; South Florida Journal of Development, Miami, v.2, n.2 , p. 3284 - 3298 apr./jun $2021 . \quad$ ISSN $2675-5459 ; \quad$ DOI: $10.46932 /$ sfjdv2n $2-165$ https://southfloridapublishing.com/ojs/index.php/jdev/article/view/445/415

Gestion.pe (2020) Padres de más de 110,000 estudiantes solicitaron traslado a colegios públicos Asignación de vacante será comunicada a los padres de familia entre el 7 y el 21 de junio. Actualizado el 23/05/2020 05:33 p.m.; Empresa Editora Gestión, Jorge Salazar Araoz N ${ }^{\circ}$ 171, La Victoria, Lima. Copyright (C) gestion.pe, Grupo El Comercio - Todos los derechos reservados https://gestion.pe/peru/padres-de-mas-de-110000-estudiantes-solicitaron-traslado-a-colegios-publicosnoticia/

Gredler, M. E., (2005) Learning and Instruction: Theory into Practice - 5th Edition, Upper Saddle River, NJ, Pearson Education.

Hernández -Sampìeri R. y Mendoza Torres C. P. (2019) Metodología de la Investigación, Las rutas cuantitativa, cualitativa y mixta

López López, G., (2015) Metales de las tierras raras; del académico de número de la Academia de Ciencias de la Región de Murcia, España, https://www.um.es/acc/metales-de-las-tierras-raras-i/

Ministerio de Educación (2016) Currículo Nacional de la Educación Básica (DCN) aprobado mediante Resolución Ministerial No 281-2016-MINEDU del 02 de junio del 2016, Calle Del Comercio Nº 193, San Borja; http://www.minedu.gob.pe/curriculo/pdf/curriculo-nacional-2016.pdf

Montoya Acosta L., Parra Castellanos M., Lescay Arias M., Cabello Alcívar O. \& Coloma Ronquillo G. (2019) en su investigación "Teorías pedagógicas que sustentan el aprendizaje con el uso de las TICs" Revista Información Científica, Volumen 98 No. 2 marzo-abril 2019 ISSN 1028-9933

Organización de las Naciones Unidas para la Educación, la Ciencia y la Cultura (UNESCO) (2020) en el documento "Aportes para una respuesta educativa frente al COVID-19 en América Latina" Análisis desde la evidencia del Laboratorio Latinoamericano de Evaluación de la Calidad de la Educación (LLECE)

Pino Salamanca Stella \& Euscátegui Pachón Robert Alfredo (2021) La educación popular una apuesta política que se recrea en los procesos educativos desde el trabajo colectivo de los educadores populares del cauca; mail: stellapino@unicauca.edu.co y raeusca@unicauca.edu.co; South Florida Journal of 
Development, Miami, v.2, n. 4 , p. 5008 - 5016 jul./sep . 2021 ISSN 2675 - 5459; DOI: 10.46932/sfjdv2n 4 - 005 https://southfloridapublishing.com/ojs/index.php/jdev/article/view/656/601

Plank, David, José Amaral Sobrinho y Antonio Carlos da Resurreiçao Xavier (1994), «Obstacles to Educational Reform in Brazil», La educación, 1, n. ${ }^{\circ}$ 117, (OEA), Washington, D.C., pp. 75-101.

Quispe Palomino Marino Rómulo \& Huamán Camillo Javier Giraldo, (2021) Competencias digitales en los docentes de educación básica del Perú Correo: mquispepal@ucvvirtual.edu.pe \& jhuamanc17@ucvvirtual.edu.pe; South Florida Journal of Development, Miami, v.2, n. 3 , p. 3890 - 3904 special edition, jul. 2021. ISSN 2675 - 5459; DOI: $10.46932 / \mathrm{sfjdv} 2 \mathrm{n} \quad 3 \quad-\quad 0 \quad 07$ https://southfloridapublishing.com/ojs/index.php/jdev/article/view/528/483

Robles Pihuave C. \& Zambrano Montes L. (2020). Prácticas académicas basadas en las nuevas tecnologías para el desarrollo de ambientes creativos de aprendizaje. Rehuso, 5(2), 50- 61. https://revistas.utm.edu.ec/index.php/Rehuso/article/view/1684

Rodríguez Alayo A. \& Cabell Rosales N. (2021) en su investigación "Importancia de la competencia digital docente en el confinamiento social” Polo del Conocimiento (Edición núm. 54) Vol. 6, No 1 Enero 2021, pp. 1091-1109 ISSN: 2550 - 682X DOI: 10.23857/pc.v6i1.2210

Rodríguez Campoverde D., Peña Holguín R. \& Stracuzzi Pastor M. (2020): “Impacto e inclusión de las TIC en los estudiantes de educación básica, retos, alcance y perspectiva", Revista Atlante Cuadernos de Educación y Desarrollo, ISSN: 1989-4155 (agosto 2020). https://www.eumed.net/rev/atlante/2020/08/inclusion-tics.html

Rodríguez-Alayo A. O. \& Cabell-Rosales N. V. (2021) Importancia de la competencia digital docente en el confinamiento social, arodriguezala@ucvvirtual.edu.pe https://orcid.org/0000-0001-8011-4013; ncabell@ucvvirtual.edu.pe, https://orcid.org/0000-0003-0019-846X; Ciencias Técnicas y Aplicadas

Siemens G. (2007) Conectivismo: Una teoría de aprendizaje para la era digital (gsiemens@elearnspace.org) Diciembre 12, 2004 Traducción: Diego E. Leal Fonseca (diego@diegoleal.org) investigador del Laboratorio de Investigación y Desarrollo sobre Informática en Educación de la Universidad de los Andes, en Bogotá, Colombia.

Socialhizo.com (2021) Historia de la Filosofía: La Escolástica Medioeval, https://www.socialhizo.com/historia/historia-de-la-filosofia-la-escolasticamedieval\#: :text=En\%20el\%20siglo\%20XIII\%20surge,Aquino\%20(1224\%2D1274).

Valdez Alejandre F. (2012) en su investigación "Teorías educativas y su relación con las tecnologías de la información y de la comunicación (TIC)" Áreas de investigación: Educación en contaduría, administración e informática, Universidad Nacional Autónoma de México, Ciudad de México, México.
Wikipedia.org
(2021)
Emisión
Termoiónica,

https://es.wikipedia.org/wiki/Emisi\%C3\%B3n_termoi\%C3\%B3nica 